\title{
IR Remote Controlled Dish Positioning System
}

\author{
Kamal Bhatia, Anjana Rani Gupta
}

\begin{abstract}
The present paper relates to a dish positioning system by a single IR remote. The system includes a microcontroller for processing commands related to positioning of the dish. The use of a dish is mainly to receive signals coming from satellite, and the aim of the project is to adjust the position of the dish for getting maximum number of signals from satellite. The manual process of doing this is very much time taking and can be incorrect also, so an automated dish positioning system is required to overcome these problem of manual process. The main components that are required for angle control of dish mainly are microcontroller, motor, and an IR remote. The signal transmitted by the remote to the IR receiver and then receiver further sends the signal to the microcontroller. Microcontroller is loaded with basic programming, therefore microcontroller controls the motor via a motor driver IC. By the implementation of automated system for controlling angle of dish, the system becomes more efficient and accurate.

Index Terms-Microcontroller, Motor driver IC, DC Motor, IR remote,
\end{abstract}

\section{INTRODUCTION}

The dish positioning system is implemented which can be controlled with help of a TV remote to set the position. The purpose of dish is receiving signals from broadcasting sources. The manual process of positioning the dish at accurate angle so that maximum value of signals are received of a specific frequency is very time consuming and difficult. To resolve the problem, there is a need to develop a system which introduces the automation in dish positioning process. In this project a system for setting the position of dish by a single TV remote is implemented. 2 motors are employed in the system for moving the dish in vertical and horizontal both the directions. The TV remote which controls the dish acts as a transmitter and the information transmitted by the remote will be received by an infrared receiver. The receiver is interlinked to an 8051 microcontroller. Basically the data transmission directs from remote to the microcontroller via receiver. The microcontroller which is interlinked to the receiver, receives signal from and then transmits control signals to the motors which are employed for moving dish.

To drive these 2 motors a motor driver IC is employed in the system. The coding is a standardized RC5 coding that is tracked by the TV remote. For recognition of input code from the TV remote this code has been used for the controller for developing suitable output signal for the motor driver IC. Advancement in the project can be introduced by employing RF control as an alternative of IR that has limitations of line of sight communication.

Revised Version Manuscript Received on 10 September, 2019.

Kamal Bhatia, Department of Electronics \& Communication Engineering, Noida Institute of Engineering and Technology, UttarPradesh, India.. (Email: researchnietip@gmail.com)

Dr. Anjana Rani Gupta, Department of Mathematics, Noida Institute of Engineering and Technology, UttarPradesh, India. (Email: researchnietip@gmail.com)
The motor which is most popular is servo motor used for controlling and driving heavy loads, and also the cost of the motor is very high for the use as explained in paper [2].In paper [1] it is disclosed that employment of remote control with the system increases the advancement of the technology, use of microcontroller positions the motor at desired angle and maintain it at that position, Though the 1st step to approach is this for controlling the system, robotics systems, and automation system[9].

The second section discusses about the problem statement, third section is block representation of the system, and fourth section explains conceptual architecture, fifth is designing, sixth section contains advantages, seventh section discloses about results and last section is conclusion.

\section{PROBLEM STATEMENT}

The system can be implemented by using computer instead of a microcontroller. The reason for using a microcontroller is that it is a single chip controller and has features like small size, low cost and high performance. Therefore, a microcontroller is appropriate for automatic positioning of dish system. A satellite locating system is established in the present system. The capability of tracking in a dish positioning system is a very crucial point. Controlling of dish manually is very much time consuming and there can be inaccuracy in the manual process. The system comprises of a remote for starting the motor and then moving it in required direction. The use of a remote control for monitoring the system makes the system advanced and employment of microcontroller moves the motor in required direction. Though for the robotics, control system, automation system this is the initial step to search and is able to assist controls in industries. 


\section{BLOCK DIAGRAM}

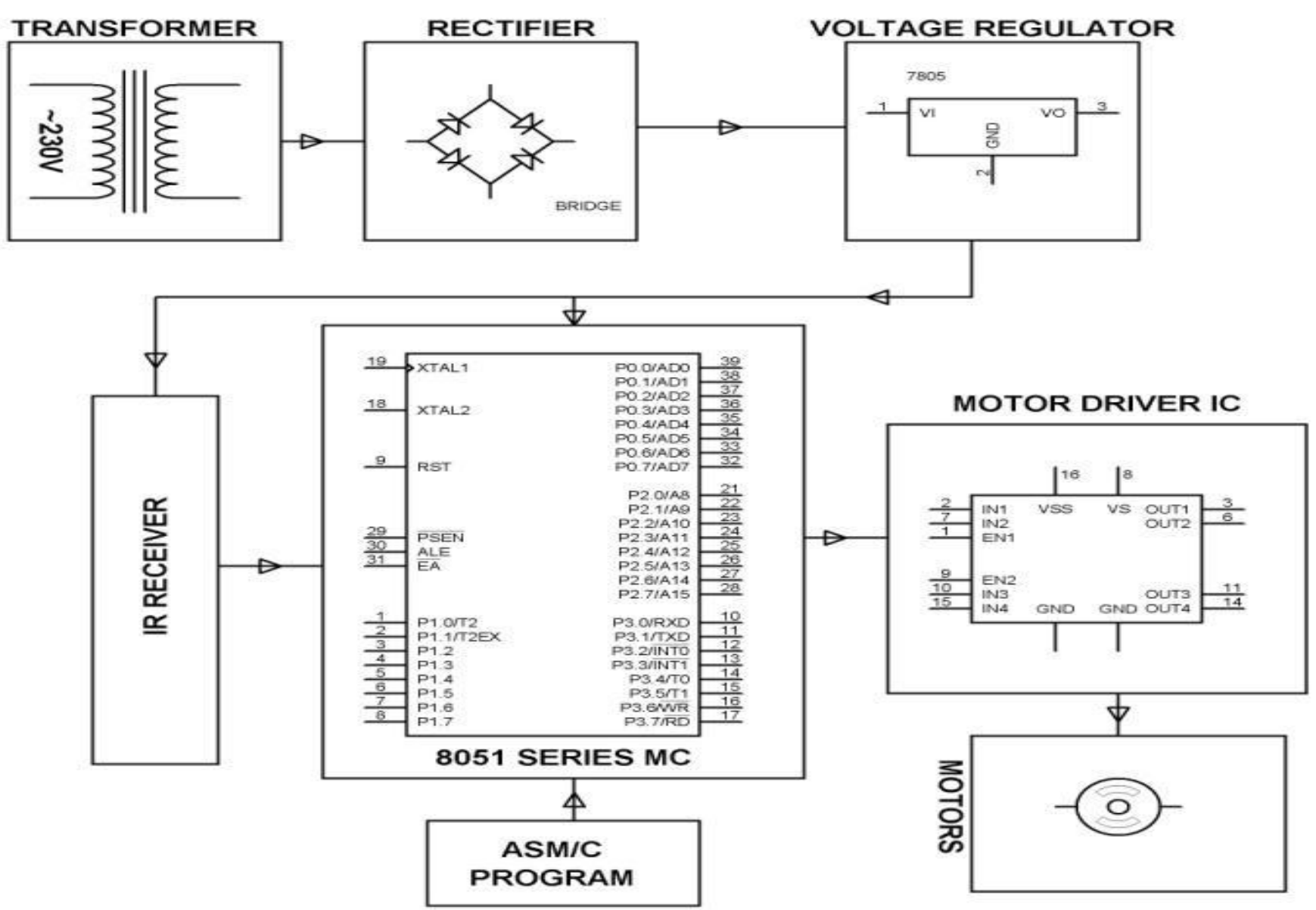

Figure 1 Architecture of embedded web server system

\section{CONCEPTUAL ARCHITECTURE}

There are 3 parts of the architecture, given as: (1) Remote environment; (2) Arduino UNO \& (3) internet connectivity in home.

Initially, when the supply power is applied to the system, then motor rotates at a random angle, known as last degree. When a switch of setting is pushed for saving the degree and degree then the motor would go from present state and stops at that position. At this position value of degree and count is zero. Then it would reach at the uppermost limit. After this, the motor is at halt position and then highest value of degree would be stored in the microcontroller, thereby highest count and degree value is detected. EEPROM is equipped with stored pulses and angle degrees and resolution is calculated by the microcontroller. When a command for angle degree by the remote controller is given to the microcontroller, the motor starts moving. The counts are driven by the, and sensors senses these counts. Sensor provides the counts to the motor by a feedback. Sensor provides these counts to the microcontroller via a feedback link. The number of counts are increased by the microcontroller when the angle command is bigger than the previous one. The motor stops while command degree is equivalent to the last degree when the counts are in increasing process. In same the way, the counts are decreased by the microcontroller when the command angle is lesser than the previous angle. When degrees become equal, microcontroller stops the motor.

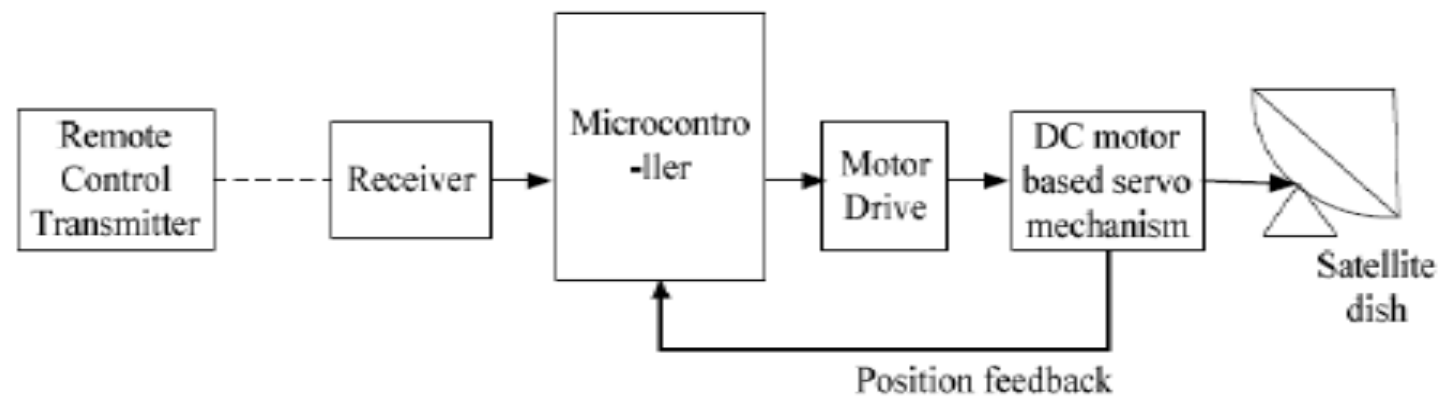

Figure 2 Architecture based on concepts 


\section{DESIGN IMPLIMENTATION}

A microcontroller is employed for driving motor. The microcontroller moves the motor in clockwise direction if the angle given in command is larger than present angle position to increase the angle and in anticlockwise path if the angle given in command is lesser than the present angle in degrees as shown in Flow Chart refer Figure 3.

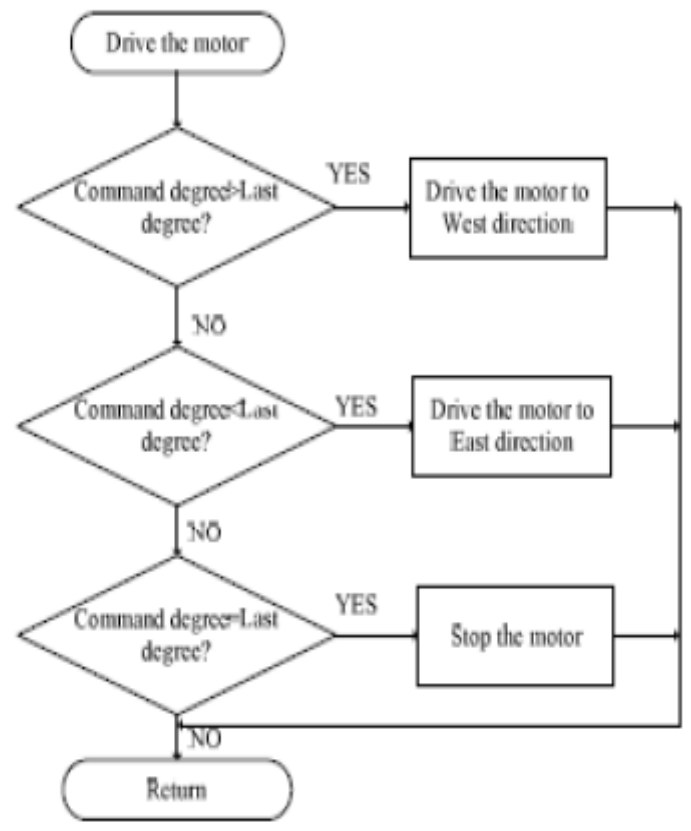

Figure 3 Flow Chart

\section{ADVANTAGES}

The position of dish i.e angle at which dish is inclined plays a very important role to get maximum number broadcasted satellite signals. The placement of dish must be at right angle for getting maximum possible signals. When positioning of dish is done manually, best position may not be directed. The presented system is planned to regulate the position of the dish by a single TV remote after pushing the buttons present on it according to the track of dish position.

\section{RESULT}

The angle of the dish has been changed with the help of remote control and a microcontroller. The perfect angle has been selected for tracking maximum number of signals.

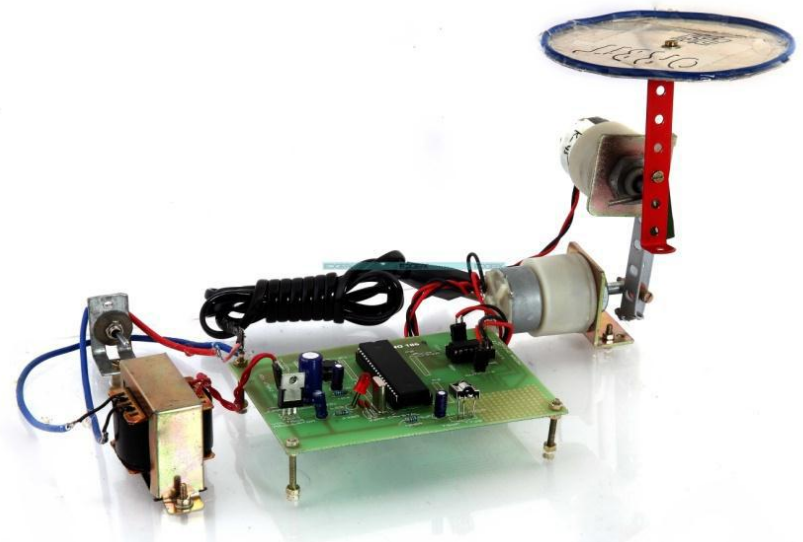

Figure 4 System diagram

\section{CONCLUSION}

A system to position a dish has been monitored by using an IR remote control successfully. The system works properly as it is positioning the dish in desired position at desired angle, thereby maximum number of signals can be trapped. The use of microcontroller in the system is most appropriate for automatic positioning systems. In the present system, a system for positioning satellite has been implemented. The system ability of tracking is at critical point. The system positions the dish with the help of a single remote and moves the motor according to requirement. Now a days microcontrollers are used in wide spread as it is compact in size and uses the latest technology. The system provides accuracy in position of dish and lessens the time consumption for positioning.

\section{REFERENCES}

1. Chatterjee S., "Industrial Electronics and Control", New Delhi: Tata McGraw-Hill Publishing Company Limited.

2. Richard, V., Editor, "Motor Control Electronics Handbook, McGraw-Hill, Boston.

3. R. Krishnan, Electric Motor Drives, Low Price Edition.

4. Nhivekar G.S. and Mudholkar R.R. "Microcontroller Based IR Remote Control Signal Decoder for Home Application".

5. Ian Aber, James Binney, Miles Buckman, Samantha Krening, Vu Nguyen, Michael Reher, and Miranda Rohlfing "Creating a Control and Operating System For Monitoring and Observing Space(COSMOS)”. 